\title{
TWEAK/Fn14 signaling mediates gastric cancer cell resistance to 5-fluorouracil via $\mathrm{NF}-\mathrm{kB}$ activation
}

\author{
OH-HYUNG KWON ${ }^{1}$, JONG HWAN KIM ${ }^{1,2}$, SEON-YOUNG KIM ${ }^{1,2}$ and YONG SUNG KIM ${ }^{1,2}$ \\ ${ }^{1}$ Medical Genomics Research Center, KRIBB; ${ }^{2}$ Department of Functional Genomics, \\ University of Science and Technology, KRIBB, Daejeon 305-806, Republic of Korea
}

Received September 26, 2013; Accepted November 18, 2013

DOI: $10.3892 /$ ijo.2013.2211

\begin{abstract}
Chemoresistance is one of the most serious problems in the treatment of cancer. In the present study, we show that Fn14 promotes resistance to 5-fluorouracil (5-FU) in gastric cancer (GC). We found that 5-FU treatment upregulated Fn14 expression in various cancer cell lines, including GC cell lines, and that knockdown of Fn14 using shRNA accelerated 5-FU sensitivity. In contrast, Fn14 overexpression or TWEAK treatment promoted resistance to 5-FU. Furthermore, we investigated the mechanisms underlying Fn14-mediated chemoresistance. We first revealed that 5-FU-mediated upregulation of Fn14 occurred as a result of NF- $\kappa \mathrm{B}$ activation, indicating that 5-FU-mediated $\mathrm{NF}-\kappa \mathrm{B}$ activation was the principal event underlying Fn14 upregulation and 5-FU resistance in GC. Taken together, our results suggest that Fn14 is a novel therapeutic target and that inhibition of Fn14 combined with 5-FU treatment may be an effective molecular therapeutic strategy to treat 5-FU-resistant gastric cancers.
\end{abstract}

\section{Introduction}

Fibroblast growth factor-inducible-14 (Fn14), originally identified as one of the growth factor-inducible genes present in murine fibroblasts, is the smallest member of the tumor necrosis factor (TNF) superfamily of receptors that lacks the cytoplasmic death domain (1-3). The Fn14 gene is expressed in many tissues, including the heart, placenta, kidneys, lungs, and pancreas, and is located on human chromosome 6 (2). In the GENT database, Fn14 is upregulated in many tumor tissues compared to normal tissues (4) and has also been reported to be elevated in various cancers, including GC $(2,5,6)$. Addition

Correspondence to: Dr Yong Sung Kim or Dr Seon-Young Kim, Medical Genomics Research Center, KRIBB, Daejeon 305-806, Republic of Korea

E-mail: yongsung@kribb.re.kr

E-mail:kimsy@kribb.re.kr

Abbreviations: 5-FU, 5-fluorouracil; GC, gastric cancer; CCK-8, cell counting kit-8

Key words: chemoresistance, 5-FU, gastric cancer, NF-кB, Fn14 of TNF-like weak inducer of apoptosis (TWEAK), a ligand for Fn14, activates Fn14 and increases cell survival, growth, and migration in some cancers $(5,6)$. Inhibition of Fn14 expression decreases migration and invasion, whereas ectopic expression of Fn14 increases the invasive activity of prostate cancer cells (7). The levels of Fn14 expression also affect the growth of GC cells (6). A recent study reported that immunotoxins targeting the Fn14 receptor induce melanoma cell necrosis, which leads to growth inhibition (8), suggesting that Fn14 may be an appropriate therapeutic target for cancer.

$\mathrm{NF}-\kappa \mathrm{B}$ is activated in response to various inflammatory agents, carcinogens, tumor promoters, and growth factors and regulates the expression of various genes that are involved in immunity, inflammation, cell survival, or oncogenesis $(9,10)$. $\mathrm{NF}-\kappa \mathrm{B}$ is an inhibitor of apoptotic cell death and activates several target genes that are involved in preventing the induction of apoptosis (11). NF- $\mathrm{KB}$-mediated anti-apoptotic factors contain cIAPs, c-FLIP, and members of the Bcl-2 family (11). $\mathrm{NF}-\kappa \mathrm{B}$ is constitutively active in cancer cells and is highly resistant to antitumor drugs or ionizing radiation, while inhibition of NF- $\kappa \mathrm{B}$ activity greatly promotes sensitivity to such treatments (12). TWEAK/Fn14 signaling is one of the important upstream stimulators of $\mathrm{NF}-\kappa \mathrm{B}$ activation resulting in induction of the expression of NF- $\mathrm{BB}$-regulated anti-apoptotic genes $(13,14)$. These anti-apoptotic genes are associated with the inhibition of apoptosis and the resistance to anticancer drugs, resulting in poor prognosis and shortened survival (15), suggesting that the TWEAK/Fn14/NF- $\kappa \mathrm{B}$ cascade may be involved in chemoresistance.

Identification of the major molecules that regulate cancer resistance and the development of new strategies to treat the disease are required to find an effective cure for cancer. Gastric cancer is a common malignancy that is associated with a high mortality rate. It is the fourth most commonly diagnosed cancer in the world and the third most common cause of cancer-related deaths (16). Although many antitumor drugs have been used to treat patients with cancer, drug resistance has become a serious problem. Overcoming the problem of drug resistance could improve the efficacy of antitumor drugs. 5-FU is a chemotherapeutic agent that is widely used for the treatment of GC. 5-FU exerts its toxic effects on cancer cells by inducing apoptosis $(17,18)$. However, the mechanism by which 5-FU activates the apoptotic process is not fully understood in GC. Clarification of the underlying mechanism may help 
to decrease the frequency of resistance to 5-FU-induced cell death in cancer. In this study, we investigated how the Fn14 molecule contributes to 5-FU chemoresistance in GC cells.

\section{Materials and methods}

Cell lines. Gastric cancer cell lines were cultured in complete RPMI-1640 medium. 293T cells were maintained in complete DMEM media. All cell lines were obtained from the Korean Cell Line Bank (http://cellbank.snu.ac.kr/index.htm), and all complete media contained 10\% fetal bovine serum (Hyclone, Logan, UT, USA), $100 \mathrm{U} / \mathrm{ml}$ of penicillin/streptomycin (Invitrogen, Carlsbad, CA, USA), $2 \mathrm{mM}$ L-glutamine, and $0.5 \mathrm{mM}$ HEPES.

Lentiviral packaging and transduction of Fn14 small hairpin $R N A$ (shRNA). A non-targeting shRNA control vector (catalog no. SHC002) and shRNA lentiviral vectors targeting human Fn14 mRNA (catalog no. TRCN0000072451) were purchased from Sigma-Aldrich. For lentivirus production, the shRNA vector was cotransfected with the lentiviral packaging mix (Sigma-Aldrich, Taufkirchen, Germany) into 293T cells using FuGENE HD (Roche Diagnostics, Indianapolis, IN, USA) according to the manufacturer's protocol. SNU-216 and MKN-1 cells were infected and selected using $1 \mu \mathrm{g} / \mathrm{ml}$ of puromycin. Fn14 protein reduction was assessed using western blot analysis.

Fn14 stable cell lines. Fn14 expression plasmids (6) were transfected into AGS cells using Lipofectamine Plus reagent (Invitrogen) according to the manufacturer's protocol. Transfected cells were cultured for 2 days prior to selection in the presence of $1 \mathrm{mg} / \mathrm{ml}$ of G418 in complete medium. Fn14 protein levels were assessed using western blot analysis.

Transfection and luciferase reporter assays. SNU-216 cells were seeded into a 24 -well plate 1 day prior to transfection. The cells were transiently transfected with the NF- $\kappa B$ luciferase reporter gene (Clontech, Palo Alto, CA, USA) using Lipofectamine Plus reagent (Invitrogen) according to the manufacturer's protocol and were treated with either various amounts of $5-\mathrm{FU}, 20 \mu \mathrm{g}$ of $5-\mathrm{FU}$ alone, or $20 \mu \mathrm{g}$ of $5-\mathrm{FU}$ in combination with $20 \mu \mathrm{M}$ PS1145 for $24 \mathrm{~h}$. Forty-eight hours after transfection, the cells were harvested, and luciferase activity was determined using the luciferase assay system (Promega, Madison, WI, USA) with a luminometer. Luciferase activities were normalized to $\beta$-galactosidase activity using the $\beta$-galactosidase enzyme assay system (Promega) according to the manufacturer's instructions.

siRNA transfection. A non-targeting siRNA control (catalog no. SN1003) and siRNAs targeting human Bcl-xL (catalog no. 1011922) or p65 mRNA (catalog no. 1128166) were purchased from Bioneer (Daejeon, Korea). For siRNA transfection, SNU-216 and MKN-1 cells were transfected with siRNAs (100 nM) using Lipofectamine Plus (Invitrogen), and the knockdown of Bcl-xL and p65 was quantified $48 \mathrm{~h}$ posttransfection using qRT-PCR and western blotting. To assess the effects of Bcl-xL and p65 siRNA on cell growth, SNU-216 and MKN-1 cells were seeded in 96-well plates and transfected with siRNAs (100 $\mathrm{nM}$ ) using Lipofectamine plus (Invitrogen). Cell growth was measured 24 and $48 \mathrm{~h}$ after 5-FU treatment using the CCK-8 reagent (Dojindo, Kumamoto, Japan).

Real-time RT-PCR. Total RNA was isolated from GC tissues using an RNeasy kit (Qiagen, Valencia, CA, USA) according to the manufacturer's instructions prior to treatment with DNase I (Promega). DNase-treated RNA was reverse transcribed using Superscript II reverse transcriptase (Invitrogen). qRT-PCR was performed using an Exicycler Quantitative Thermal Block (Bioneer). The resulting cDNA was amplified using 2X SYBR Premix EX Taq (Takara, Shiga, Japan). $\beta$-actin was used as a loading control. The fold change in the expression of each gene was calculated using the $\Delta \Delta \mathrm{Ct}$ method (19). The primer sequences used were as follows: human p65, 5'-GCGAGAGGAGCACAGATACC-3' (forward) and 5'-CTGATAGCCTGCTCCAGGTC-3' (reverse); human Fn14, 5'-TTT CTG GCT TTT TGG TCT GG-3' (forward) and 5'-CTT GTG GTT GGA GGA GCT TG-3' (reverse); Bcl-xL, 5'-CTG AAT CGG AGA TGG AGA CC-3' (forward) and 5'-TGG GAT GTC AGG TCA CTG AA-3' (reverse); and human $\beta$-actin, 5'-CAA GAG ATG GCC ACG GCT GCT-3' (forward) and 5'-TCC TTC TGC ATC CTG TCG GCA-3' (reverse).

Western blot analyses. The cells were pelleted and lysed in NP-40 lysis buffer $(20 \mathrm{mM}$ Tris-HCl, $\mathrm{pH} 8.0,140 \mathrm{mM}$ $\mathrm{NaCl}, 10 \%$ glycerol, $1 \%$ NP-40, 2 mM EDTA, and protease inhibitor). Protein levels were quantified using the Bio-Rad protein assay method. The cell lysates were separated using SDS-PAGE. After electrophoresis, the proteins were transferred to nitrocellulose membranes, blotted with the relevant antibodies, and detected using ECL reagent (Amersham Bioscience, Buckinghamshire, UK). The primary antibodies used included Fn14 (Cell Signaling, Beverly, MA, USA), Bcl-xL (Cell Signaling), p65 (Santa Cruz Biotechnology, Santa Cruz, CA, USA), phosphor-IкB (Cell Signaling), IкB (Santa Cruz Biotechnology), and $\alpha$-tubulin (Sigma).

Caspase-3/7 assay. SNU-216 and MKN-1 cells were seeded in 96-well plates and treated with 5-FU. After $48 \mathrm{~h}$ of treatment, the cells were washed once with $1 \mathrm{X}$ PBS, trypsinized, washed again with $1 \mathrm{X}$ PBS, and then resuspended in PBS. The cells were mixed with caspase-Glo-3/7 reagent (Promega) and incubated for $1 \mathrm{~h}$ at room temperature with agitation. The luciferase activity was measured using a luminometer and then normalized according to protein concentration.

Cell growth assay. Cells from different groups were plated into 96 -well plates at $1 \times 10^{4}$ cells/well and maintained at $37^{\circ} \mathrm{C}$ in a humidified incubator. The cells were treated with various amounts of 5-FU for $48 \mathrm{~h}$. To assess the effect of TWEAK on cell survival, the cells were treated with $50 \mu \mathrm{g}$ (SNU-216) or $20 \mu \mathrm{g}$ (MKN-1) of 5-FU alone or in combination with TWEAK $(100 \mathrm{ng} / \mathrm{ml})$ for $24 \mathrm{~h}$. Cell growth was measured using the cell counting kit (CCK)-8 (Dojindo).

Statistical analyses. Statistical analyses of differences between groups were performed using Student's t-test. A P $<0.05$ was considered to be significant. 
A

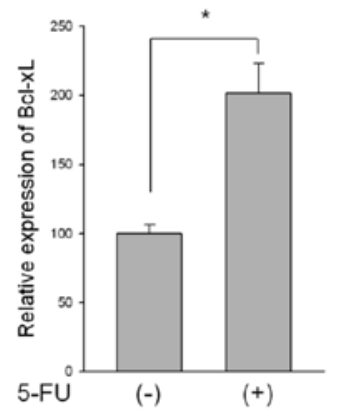

C

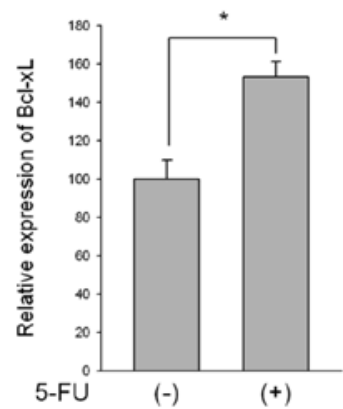

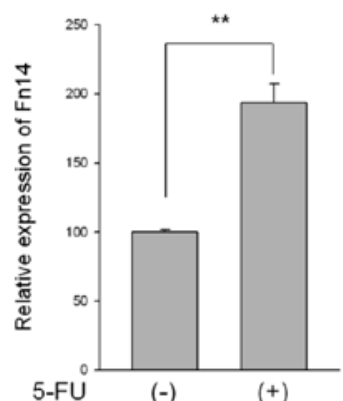

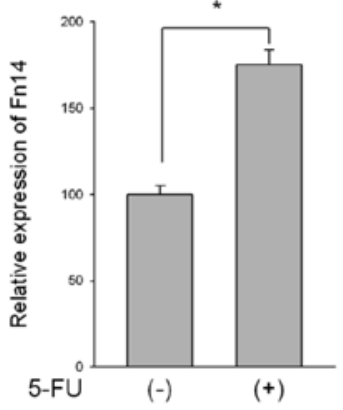

B

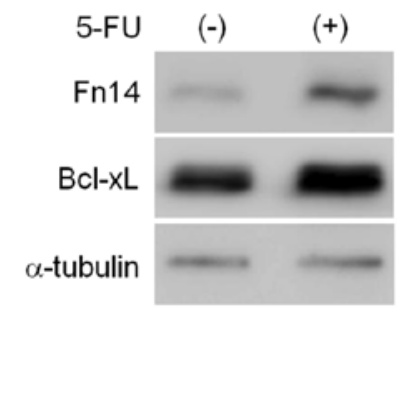

D

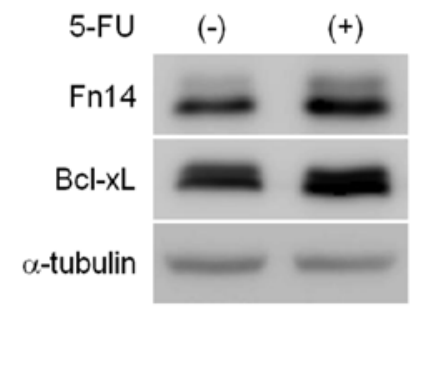

Figure 1. 5-FU-mediated upregulation of Fn14 gene expression in gastric cancer cells. (A) qRT-PCR and (B) western blot analysis of Fn14 and Bcl-xL expression in SNU-216 cells. (C) qRT-PCR and (D) western blot analysis of Fn14 and Bcl-xL expression in MKN-1 cells. The cells were treated with 50 $\mu \mathrm{g}$ of 5 -FU (SNU-216) or $20 \mu \mathrm{g}$ of 5-FU (MKN-1) for $48 \mathrm{~h}$. Total RNA and lysates were used in the qRT-PCR and western blot assays, respectively. ${ }^{*} \mathrm{P}<0.05$, ${ }^{* *} \mathrm{P}<0.01$. Columns, results of triplicate experiments; error bars, SD.

\section{Results}

5-FU treatment upregulates the levels of Fn14 mRNA and protein in gastric cancer cells. NF- $\kappa \mathrm{B}$ is known to be activated by 5 -FU, resulting in increased resistance to this drug (20), and the Fn14 promoter is known to contain NF- $\kappa \mathrm{B}$ binding sites, allowing for positive feedback between Fn14 and NF- $\mathrm{BB}$ to occur (21). The results of our previous studies also revealed that Fn14 overexpression increased NF- $\kappa \mathrm{B}$ transcriptional activity in gastric cancer cells (6). Therefore, we investigated whether Fn14 was involved in chemoresistance in general and in 5-FU resistance in particular. We first examined Fn14 expression after 5-FU treatment of gastric cancer cells. Treatment with 5-FU upregulated Fn14 expression at both the mRNA and protein levels in SNU-216 and MKN-1 cells (Fig. 1). We also examined Bcl-xL expression because $\mathrm{Bcl}-\mathrm{xL}$ is regulated by Fn14 and NF- $\kappa \mathrm{B}(6)$ and the expression of $\mathrm{Bcl}-\mathrm{xL}$ is induced by 5-FU. Bcl-xL is also known to be upregulated in 5-FU-resistant colon cancer cells (22). As predicted, Bcl-xL was upregulated following 5-FU treatment in both cell lines (Fig. 1).

5-FU upregulates Fn14 gene expression via $N F-\kappa B$ activation. We investigated the underlying mechanism by which Fn14 gene expression was upregulated following 5-FU treatment. We first performed NF- $\kappa \mathrm{B}$ luciferase assays to examine 5-FU-mediated alterations in $\mathrm{NF}-\kappa \mathrm{B}$ transcriptional activity. $5-\mathrm{FU}$ treatment increased the activity of the $\mathrm{NF}-\kappa \mathrm{B}$ reporter gene in a dose-dependent manner (Fig. 2A). We further confirmed that inhibition of NF- $\kappa \mathrm{B}$ activity was increased by 5-FU using the I $\kappa \mathrm{B}$ kinase (IKK) inhibitor PS1145 (Fig. 2B). We then inspected the expression levels of Fn14 after treatment of the cells with 5-FU alone or in combination with PS1145. As a result of 5-FU treatment, the increased levels of Fn14 expression were significantly reduced by PS1145 treatment at both the mRNA and protein levels (Fig. 2C and D). Bcl-xl also displayed similar results to those observed for Fn14 (Fig. 2C and D). In an effort to confirm the effects of NF- $\kappa$ B on Fn14 expression following 5-FU treatment, we used p65 siRNA to directly inhibit p65 expression. Similar to the results obtained following PS1145 treatment, knockdown of p65 reduced the increased expression levels of Fn14 as well as Bcl-xL following 5-FU treatment (Fig. 2E and F), indicating that the 5-FU-mediated upregulation of Fn14 expression occurred as a result of $\mathrm{NF}-\kappa \mathrm{B}$ activation. We further examined the relationship between the expression levels of p65 and Fn14, as well as those of p65 and Bcl-xL, using the GENT database in gastric cancer cell lines and tissues (4). We observed significant positive correlations between the expression levels of those genes in gastric cancer cell lines and tissues (Fig. 3), further supporting the notion that strong connections between these genes exist in gastric cancer.

Fn14 restrains 5-FU-induced apoptosis. To investigate whether Fn14 expression had an effect on 5-FU-induced cell death, we transduced Fn14 shRNA into SNU-216 and MKN-1 cells using a lentiviral system. Transduction with Fn14 
A

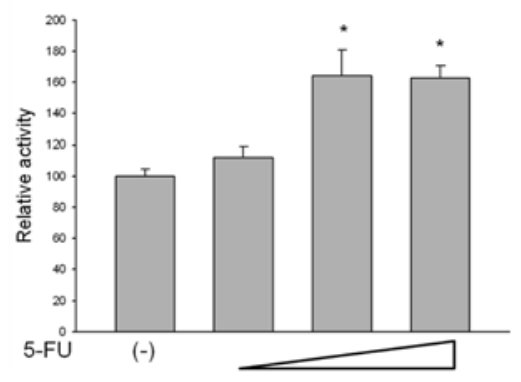

B

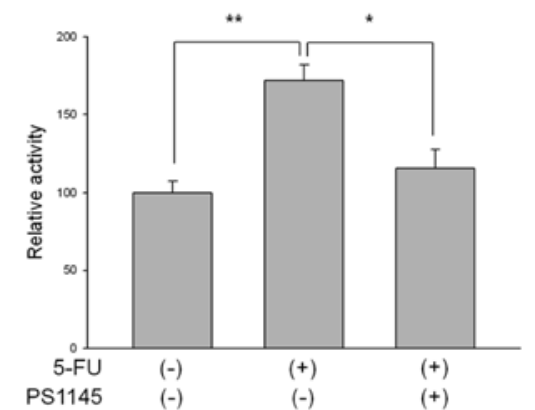

$\mathrm{E}$

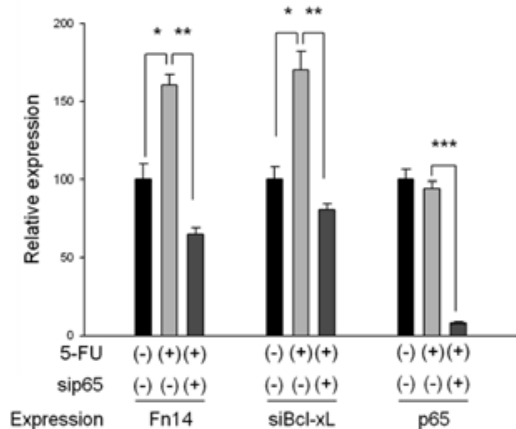

C

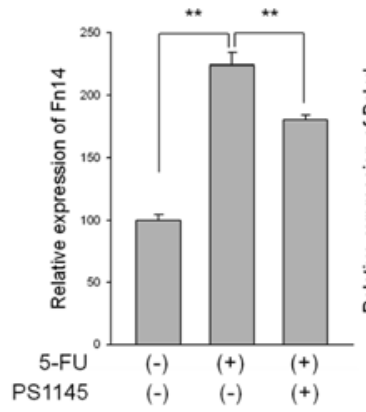

D

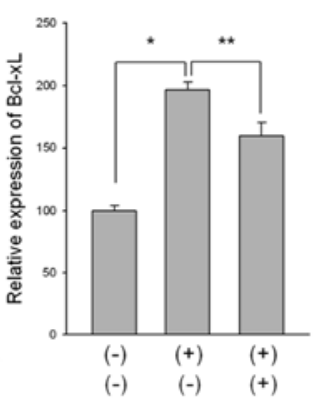

$\mathrm{F}$

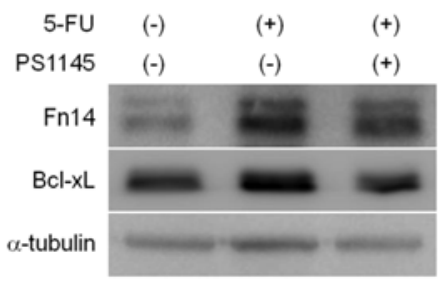

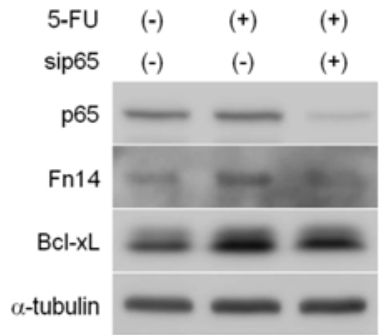

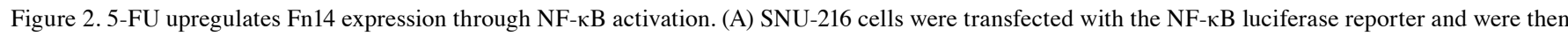
treated with 10,20 , or $50 \mu \mathrm{g}$ of 5-FU for $24 \mathrm{~h}$. The promoter activity was expressed as luciferase units relative to the activity obtained from the untreated cells (set to a value of 100). (B) SNU-216 cells were transfected with the NF-кB luciferase reporter and then treated with $20 \mu \mathrm{g}$ of 5-FU alone or in combination with $20 \mu \mathrm{M}$ PS1145 for $24 \mathrm{~h}$. (C) qRT-PCR and (D) western blot analysis of Fn14 and Bcl-xL expression in SNU-216 cells. The cells were treated with $20 \mu \mathrm{g}$ of 5-FU alone or in combination with $20 \mu \mathrm{M}$ PS1145 for $48 \mathrm{~h}$. Total RNA and lysates were used in qRT-PCR and western blot assays, respectively. (E) qRT-PCR and (F) western blot analysis of Fn14, Bcl-xL and p65 expression in MKN-1 cells. The cells were transfected with p65 siRNA and were treated with $20 \mu \mathrm{g}$ of 5-FU for $48 \mathrm{~h}$. Total RNA and lysates were used in qRT-PCR and western blot assays, respectively. ${ }^{*} \mathrm{P}<0.05,{ }^{* * *} \mathrm{P}<0.01,{ }^{* * *} \mathrm{P}<0.001$. Columns, results of triplicate experiments; error bars, SD.

A

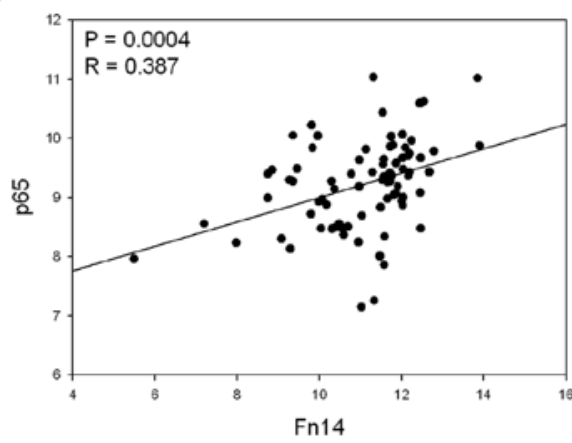

C

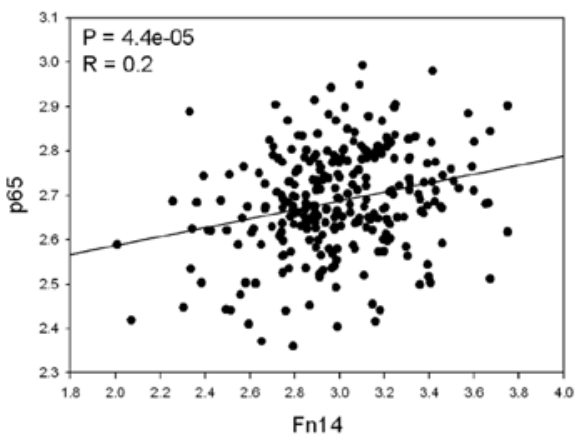

B

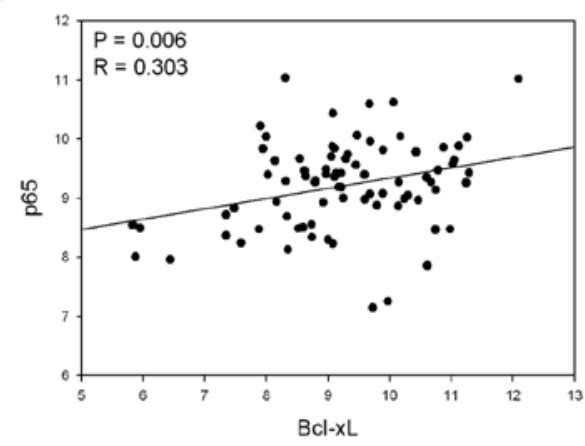

D

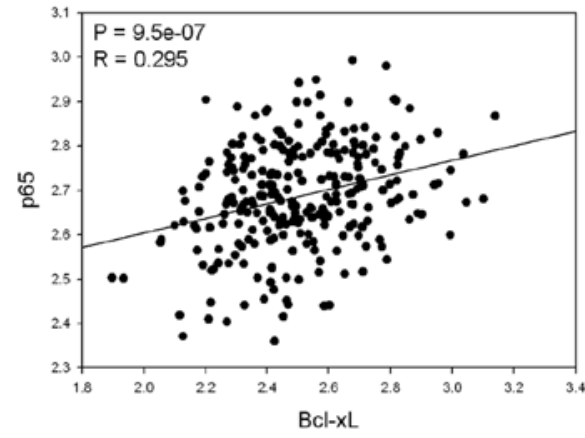

Figure 3. Comparison of the expression levels using the GENT database. Correlation between the expression levels of p65 and Fn14 (A) or p65 and Bcl-xL (B) in gastric cancer cell lines. Correlation between the expression levels of p65 and Fn14 (C) or p65 and Bcl-xL (D) in normal gastric tissues and cancer tissues. 


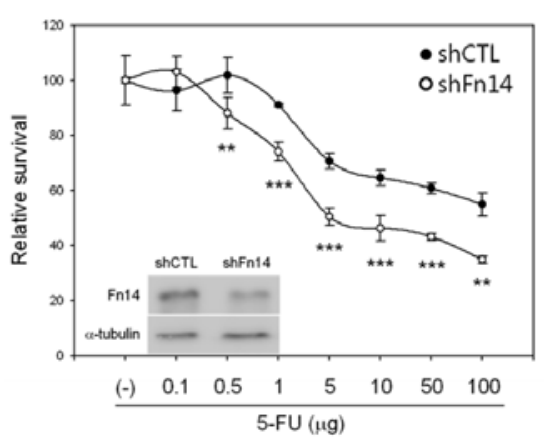

D

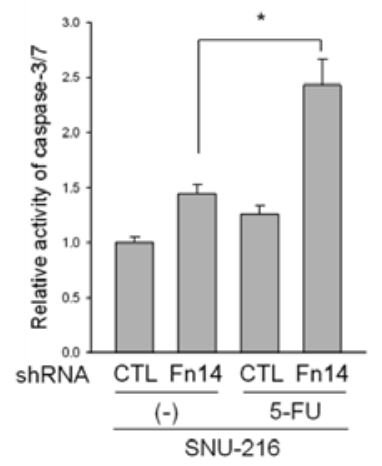

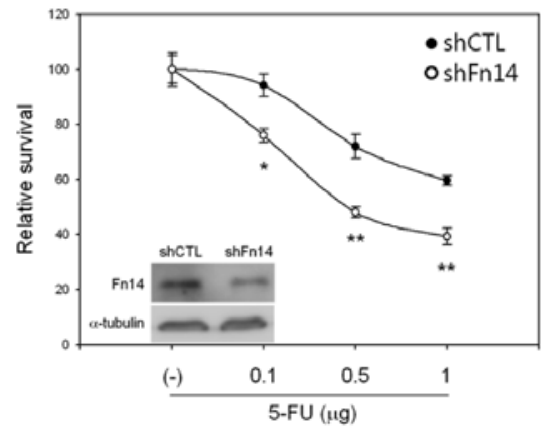

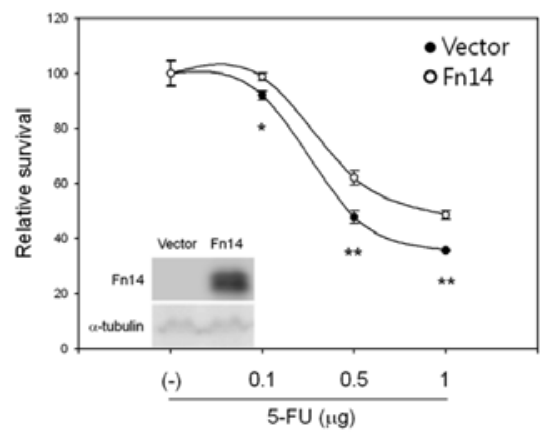

E

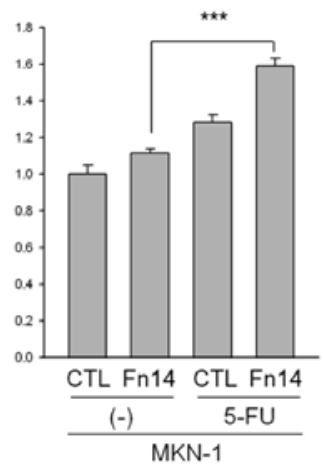

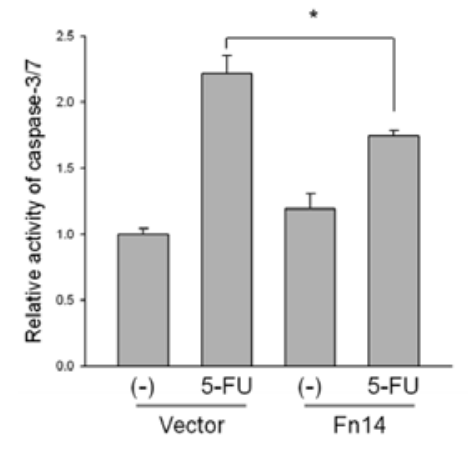

Figure 4. Fn14 expression levels affect 5-FU efficacy in gastric cancer cells. (A) SNU-216 and (B) MKN-1 cells were transduced with either Fn14 shRNA (shFn14) or control shRNA (shCTL) and were seeded in 96-well plates. Cell survival was analyzed using the CCK-8 reagent after a 48-h exposure to the indicated doses of 5-fluorouracil. (C) The survival of Fn14-overexpressing AGS cells was analyzed as in (A) and (B). (D) Plot of caspase-3/7 activity normalized to protein concentration in SNU-216 and MKN-1 cells that were grown for $48 \mathrm{~h}$ after the addition of 5 -FU (50 $\mu \mathrm{g} / \mathrm{ml}$ for SNU-216 cells and $20 \mu \mathrm{g} / \mathrm{ml}$ for MKN-1 cells, respectively). (E) Plot of caspase-3/7 activity normalized to protein concentration in AGS cells that were grown for $48 \mathrm{~h}$ after the addition of $50 \mu \mathrm{g}$ of 5-FU. ${ }^{*} \mathrm{P}<0.05,{ }^{* *} \mathrm{P}<0.01,{ }^{* * * *} \mathrm{P}<0.001$. Columns, results of triplicate experiments; error bars, $\mathrm{SD}$.

shRNA resulted in reduced levels of Fn14 protein compared to transduction with control shRNA (Fig. 4A and B). We then examined whether reduced levels of Fn14 expression promoted sensitivity to 5-FU. As shown in Fig. 3A and B, the reduction of Fn14 expression by shRNA accelerated 5-FU-mediated inhibition of cell growth in both SNU-216 and MKN-1 cells. In contrast, overexpression of Fn14 by stable Fn14 DNA transfection in ASG cells weakened 5-FU-mediated inhibition of cell growth (Fig. 4C). We next exposed the cells to 5-FU and analyzed caspase-3/7 activity using caspase-3/7 reagent. As shown in Fig. 4D, downregulation of Fn14 significantly increased caspase-3/7 activity compared to control shRNA. In contrast, overexpression of Fn14 decreased caspase-3/7 activity compared to empty vector-transfected cells (Fig. 4E), indicating that Fn14 is an important determinant of apoptotic sensitivity following 5-FU treatment in these cells.

TWEAK stimulation also protects gastric cancer cells against 5-FU-induced apoptosis. TWEAK transactivates the Bcl-xL promoter via $\mathrm{NF}-\kappa \mathrm{B}$ activation and increases resistance to cytotoxic therapy-induced apoptosis in glioma cells (23). Therefore, we treated cells with 5-FU alone or in combination with TWEAK and then examined the effects of TWEAK on 5-FU-induced cell death. TWEAK treatment increased the rate of cell survival compared to 5-FU treatment alone in both SNU-216 and MKN-1 cells (Fig. 5A). Although Fn14 is known to be a TWEAK receptor, TWEAK can mediate the differentiation of RAW264.7 cells, which do not express Fn14, into osteoclasts, indicating that other TWEAK receptors exist in cells (24). To investigate whether these effects of TWEAK on 5-FU resistance were mediated through the Fn14 receptor, we treated cells expressing normal (shCTL) or reduced levels of Fn14 (shFn14) with 5-FU alone or in combination with TWEAK and then examined the rates of cell survival. TWEAK had no effect on 5-FU-induced apoptosis in shFn14transduced cells compared to control cells (Fig. 5B).

The presumption of NF- $\kappa$ B involvement in TWEAK/Fn14mediated resistance to 5-FU was ascertained using p65 siRNA. We first transiently transfected p65 siRNA into SNU-216 and MKN-1 cells and confirmed the reduced levels of p65 expression at the protein level via western blotting in both cell lines (Fig. 5C). The survival rate of p65 siRNA-transfected cells was compared with control siRNA-transfected cells after 5-FU treatment. The results indicated that knock-down of p65 promoted sensitivity to 5-FU (Fig. 5C). We then examined the involvement of $\mathrm{Bcl}-\mathrm{xL}$ in 5-FU resistance because $\mathrm{Bcl}-\mathrm{xL}$ is a target gene of $\mathrm{NF}-\kappa \mathrm{B}$ as well as Fn14, as mentioned previously. Knock-down of Bcl-xL using siRNA also accelerated 5-FU sensitivity compared to control siRNA (Fig. 5D).

Bcl-xL and Fn14 are also upregulated by 5-FU in various cancer cell lines. We wondered whether the upregulation of 
A

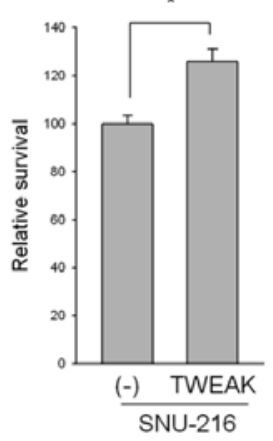

C

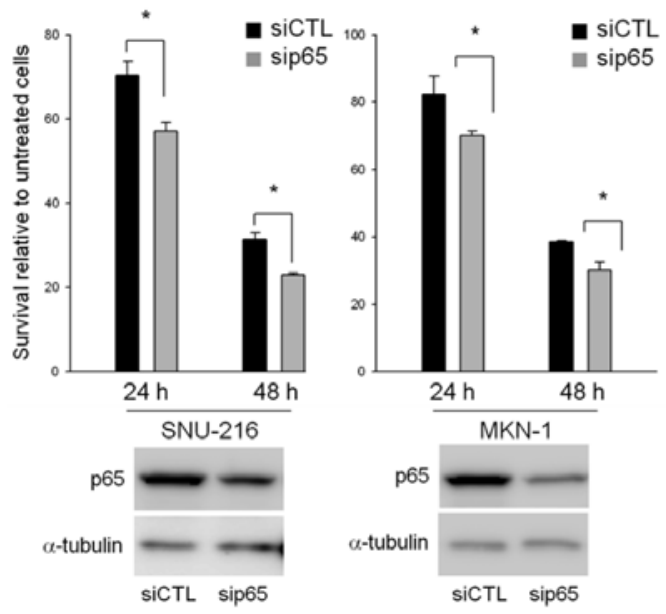

B
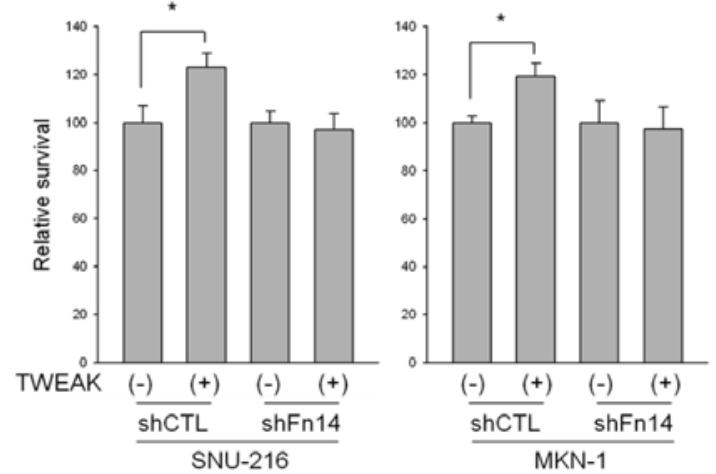

D
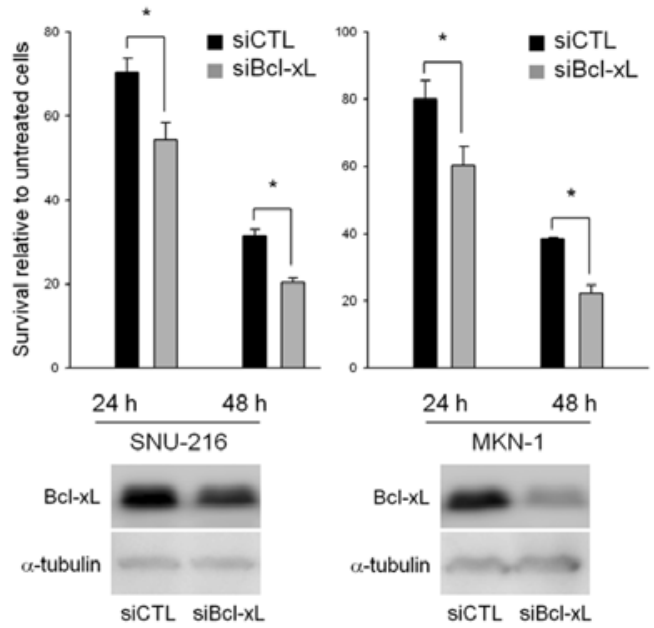

Figure 5. TWEAK promotes 5-FU resistance in gastric cancer cells. (A) SNU-216 or MKN-1 cells were treated with 50 or $20 \mu \mathrm{g}$ of 5-FU for 24 h, respectively. The serum conditions were reduced to $0.1 \%$ for $16 \mathrm{~h}$, and the cells were pretreated for $6 \mathrm{~h}$ with TWEAK (100 ng/ml) prior to 5 -FU addition. Cell survival was analyzed using the CCK-8 reagent. The growth of 5-FU-treated cells is expressed relative to untreated controls. (B) Cell survival was analyzed as in (A) in control shRNA (shCTL) or Fn14 shRNA (shFn14)-expressing cells. (C) SNU-216 or MKN-1 cells were transfected with p65 siRNA, and p65 expression was analyzed using western blotting. The cells were seeded in 96-well plates and transfected with $100 \mathrm{nM}$ concentrations of the siRNAs. Cell survival was measured 24 and $48 \mathrm{~h}$ after 5-FU treatment (50 $\mu \mathrm{g} / \mathrm{ml}$ for SNU-216 and $20 \mu \mathrm{g} / \mathrm{ml}$ for MKN-1 cells, respectively). (D) SNU-216 or MKN-1 cells were transfected with Bcl-xL siRNA, and Bcl-xL expression was analyzed using western blotting. Cell survival was measured as in (C). ${ }^{*} \mathrm{P}<0.05$, ${ }^{* *} \mathrm{P}<0.01$. Columns, results of triplicate experiments; error bars, SD.

Fn14 expression by 5-FU was limited to gastric cancer cells. To investigate whether the effects of 5-FU on Fn14 expression occurred in other cancer cell lines, we downloaded gene expression microarray data from the NCBI Gene Expression Omnibus (GEO) and examined the expression of Fn14 in various cancer cell lines. We discovered that various cell lines displayed Bcl-xL and Fn14 upregulation after 5-FU treatment (Fig. 6). Interestingly, Fn14 expression was upregulated by 5 -FU treatment in both solid (Fig. 6A and B) and liquid (Fig. 6C) tumor cells, indicating that the relationship between Fn14 and Bcl-xL expression and 5-FU is universal in cancer.

\section{Discussion}

$\mathrm{NF}-\kappa \mathrm{B}$ plays an important role in cell proliferation, apoptosis, and resistance to antitumor agents $(25,26)$. In the field of chemotherapy, persistent activation of $\mathrm{NF}-\kappa \mathrm{B}$ interrupts the effects of antitumor drugs, resulting in increased resistance to these agents (27). Therefore, the regulation of $\mathrm{NF}-\kappa \mathrm{B}$ activity may be a useful therapeutic strategy for treating cancer because drug resistance is the major reason for cancer therapy failure. We previously reported that Fn14 is involved in the growth of gastric cancer cells through $\mathrm{NF}-\kappa \mathrm{B}$ activation, leading to upregulation of the anti-apoptotic protein $\mathrm{Bcl}-\mathrm{xL}$, which is an anti-apoptotic member of the Bcl-2 family that is involved in cancer chemoresistance (28), and that high levels of Fn14 expression are associated with poor clinical outcomes among gastric cancer patients (6). These results prompted us to examine the relationship between Fn14 and NF- $\kappa \mathrm{B}$ in chemoresistance in gastric cancer. Although $\mathrm{NF}-\kappa \mathrm{B}$ is widely known to be involved in chemoresistance in cancer, additional studies are necessary to clarify the underlying mechanism by which $\mathrm{NF}-\kappa \mathrm{B}$ contributes to chemoresistance in cancer in general and in gastric cancer in particular.

5-FU-induced apoptosis is mediated predominantly by the mitochondrial apoptotic pathway, which occurs as a result of the activation of cytochrome $c$-mediated caspase-3 $(17,18)$. Evidence indicates that management of the mitochondrial apoptotic pathway may, at least in part, contribute to solving the problem of drug resistance. Bcl-2 and Bcl-xL block the release of cytochrome $c$ followed by caspase- 3 activation through stabilization of mitochondrial membrane integrity and 
A

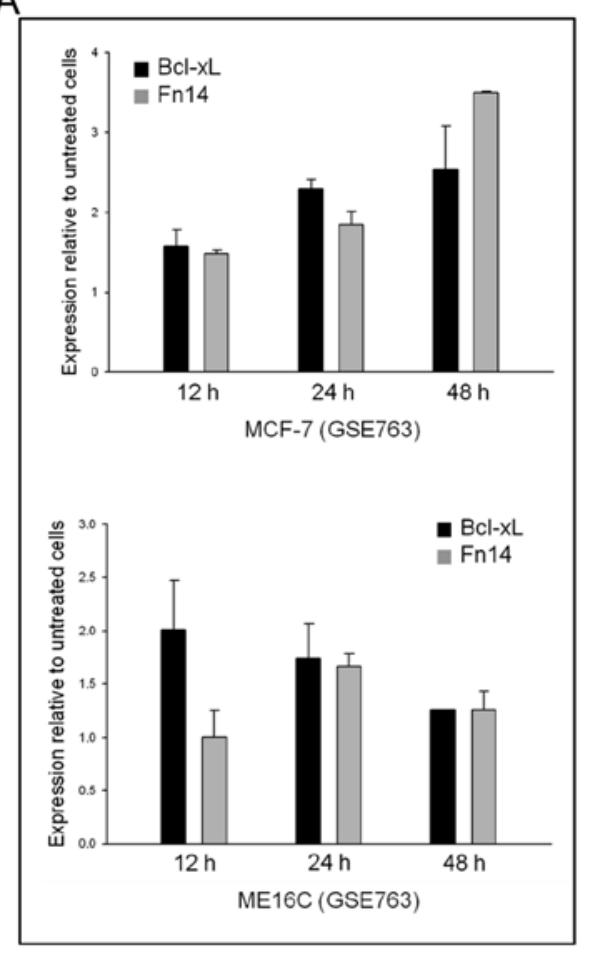

B

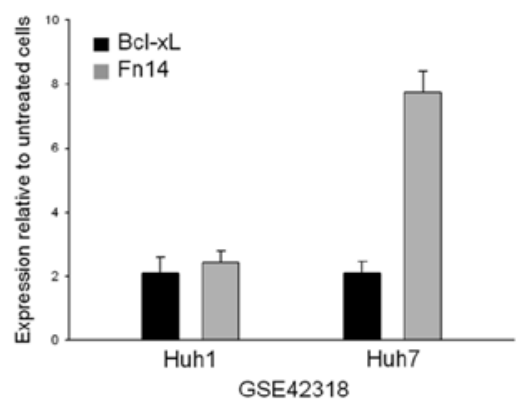

C

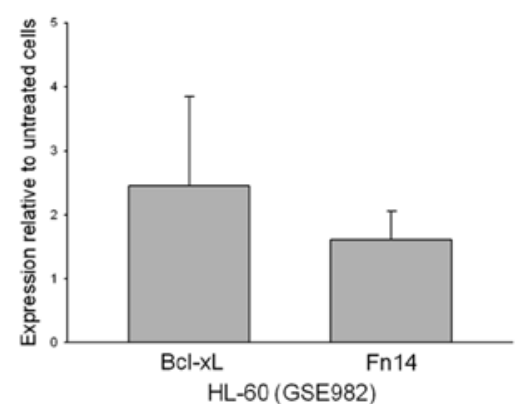

Figure 6. 5-FU upregulates Fn14 and Bcl-xL expression in various cancer cell lines. (A) Bcl-xL and Fn14 expression in breast cancer cell lines. (B) Bcl-xL and Fn14 expression in liver cancer cell lines. (C) Bcl-xL and Fn14 expression in an acute myeloid leukemia (AML) cell line. All expression levels are shown relative to untreated cells (set to a value of 1.0).

subsequent regulation of the mitochondrial apoptotic pathway $(18,22)$. Indeed, Bcl-xL is overexpressed in 5-FU-resistant cells and knock-down of Bcl-xL expression promotes sensitivity to 5-FU in colon cancer (29). In addition, Bcl-xL shows anti-apoptotic effects on other antitumor drug (30). Consistent with this evidence, our present study showed that Bcl-xL was upregulated by 5-FU and that knock-down of Bcl-xL hastened the 5-FU-induced growth inhibition of gastric cancer cells, indicating that $\mathrm{Bcl}-\mathrm{xL}$ is one of the main determinants in chemoresistance.

A previous study demonstrated that TWEAK prevents glioma cells from TRAIL- or camptothecin-induced cell death through activation of the NF- $\kappa \mathrm{B}$ pathway and that TWEAK increased the expression of anti-apoptotic proteins (23). A recent report also showed that Fn14 expression correlates with p-EGFR levels and is upregulated in EGFR-mutant cells that contain an EGFR TKI-resistant mutation (31), indicating that the TWEAK/Fn14 pathway is involved in resistance to antitumor drugs. In our present study, we showed that knock-down or overexpression of Fn14 led to the promotion of sensitivity or resistance to 5-FU, respectively. The effects of Fn14 on antitumor drugs are likely related to activation of the $\mathrm{NF}-\kappa \mathrm{B}$ pathway. NF- $\kappa \mathrm{B}$ is widely known to be regulated by TWEAK/ Fn14 signaling, indicating that TWEAK/Fn14 signaling may contribute to constitutive NF- $\mathrm{NB}$ activity in gastric cancer cells and, hence, may lead to resistance to multiple antitumor drugs.

The HER 2 proto-oncogene, which is a transmembrane tyrosine kinase receptor and a member of the ErbB protein family, is considered to be a putative Fn14 inducer. Expression of HER2 is significantly associated with that of Fn14 in breast cancer (32). Although it is not clear whether HER2 directly regulates Fn14 expression, it is of interest to note that HER2 can activate NF- $\kappa \mathrm{B}$ (33) and, hence, may lead to upregulation of Fn14 gene expression. HER-2-overexpressing breast cancer cells are less responsive to antitumor drugs than cells that normally express HER-2, and ectopic expression of HER-2 promotes resistance to chemotherapies $(34,35)$, suggesting that activation of the NF- $\mathrm{B} / \mathrm{Fn} 14$ cascade by HER-2 may be involved in chemoresistance. Fn14 activation is also elicited by proinflammatory cytokines, including IFN- $\gamma$ and IL- $1 \beta$, and treatment with the HMG-CoA reductase inhibitor atorvastatin, which is known to decrease $\mathrm{NF}-\kappa \mathrm{B}$ activation, diminishes cytokine-induced Fn14 expression in vascular smooth muscle cells $(36,37)$. In addition, increased secretion of IL- $1 \beta$ by nitric oxide (NO) leads to the induction of chemoresistance in pancreatic carcinoma cells (38). Our study using public data revealed that 5-FU treatment upregulated Fn14 expression in various cancer cell lines independent of tumor type (solid or liquid tumor). Taken together, these results strongly suggest that Fn14 may be involved in chemoresistance in many cancers as an up- or down-stream regulator.

In conclusion, we revealed that Fn14 and Bcl-xL were upregulated by 5-FU-mediated $\mathrm{NF}-\kappa \mathrm{B}$ activation and promoted resistance to 5-FU in gastric cancer cells. In addition, we demonstrated that Fn14 is a new facilitative determinant in $\mathrm{NF}-\kappa \mathrm{B}$-mediated resistance to 5-FU in gastric cancer. Positive feedback between TWEAK, Fn14, and NF- $\kappa$ B may give rise to more synergistic restraints on chemotherapy. Although it is necessary for us to elucidate whether Fn14 only limits the function of 5-FU in gastric cancer, the results presented here 
support the notion that Fn14 targeting may elevate the efficacy of current GC therapies. These results may also apply to other cancers because we discovered that Fn14 is upregulated by 5 -FU in various cancer cell lines.

\section{Acknowledgements}

This study was supported by grants from the Genomics Research Program (2012M3A9D1054670), the Future-Based Technology Development Program (NRF2011-0015710), and the KRIBB Research Initiative Program that are funded by the Korean Ministry of Education, Science and Technology.

\section{References}

1. Meighan-Mantha RL, Hsu DK, Guo Y, et al: The mitogeninducible Fn14 gene encodes a type I transmembrane protein that modulates fibroblast adhesion and migration. J Biol Chem 274: 33166-33176, 1999.

2. Feng SL, Guo Y, Factor VM, et al: The Fn14 immediate-early response gene is induced during liver regeneration and highly expressed in both human and murine hepatocellular carcinomas. Am J Pathol 156: 1253-1261, 2000.

3. Wiley SR, Cassiano L, Lofton T, et al: A novel TNF receptor family member binds TWEAK and is implicated in angiogenesis. Immunity 15: 837-846, 2001.

4. Shin G, Kang TW, Yang S, Baek SJ, Jeong YS and Kim SY: GENT: gene expression database of normal and tumor tissues. Cancer Inform 10: 149-157, 2011.

5. Tran NL, McDonough WS, Donohue PJ, et al: The human Fn14 receptor gene is up-regulated in migrating glioma cells in vitro and overexpressed in advanced glial tumors. Am J Pathol 162: $1313-1321,2003$

6. Kwon OH, Park SJ, Kang TW, et al: Elevated fibroblast growth factor-inducible 14 expression promotes gastric cancer growth via nuclear factor-kappaB and is associated with poor patient outcome. Cancer Lett 314: 73-81, 2012.

7. Huang M, Narita S, Tsuchiya N, et al: Overexpression of Fn 14 promotes androgen-independent prostate cancer progression through MMP-9 and correlates with poor treatment outcome. Carcinogenesis 32: 1589-1596, 2011.

8. Zhou H, Ekmekcioglu S, Marks JW, et al: The TWEAK receptor Fn14 is a therapeutic target in melanoma: immunotoxins targeting Fn14 receptor for malignant melanoma treatment. J Invest Dermatol 133: 1052-1062, 2013.

9. Silverman $\mathrm{N}$ and Maniatis T: NF-kappaB signaling pathways in mammalian and insect innate immunity. Genes Dev 15 2321-2342, 2001.

10. Karin M: Nuclear factor-kappaB in cancer development and progression. Nature 441: 431-436, 2006.

11. Karin M and Lin A: NF-kappaB at the crossroads of life and death. Nat Immunol 3: 221-227, 2002.

12. Wang CY, Cusack JC Jr, Liu R and Baldwin AS Jr: Control of inducible chemoresistance: enhanced anti-tumor therapy through increased apoptosis by inhibition of NF-kappaB. Nat Med 5: 412-417, 1999.

13. Saitoh T, Nakayama M, Nakano H, Yagita H, Yamamoto N and Yamaoka S: TWEAK induces NF-kappaB2 p100 processing and long lasting NF-kappaB activation. J Biol Chem 278: 36005-36012, 2003.

14. Sevilla L, Zaldumbide A, Pognonec P and Boulukos KE: Transcriptional regulation of the bcl-x gene encoding the antiapoptotic Bcl-xL protein by Ets, Rel/NFkappaB, STAT and AP1 transcription factor families. Histol Histopathol 16: 595-601, 2001.

15. Johnstone RW, Ruefli AA and Lowe SW: Apoptosis: a link between cancer genetics and chemotherapy. Cell 108: 153-164, 2002.

16. Jemal A, Bray F, Center MM, Ferlay J, Ward E and Forman D: Global cancer statistics. CA Cancer J Clin 61: 69-90, 2011.

17. Sun Y, Tang XM, Half E, Kuo MT and Sinicrope FA: Cyclooxygenase-2 overexpression reduces apoptotic susceptibility by inhibiting the cytochrome c-dependent apoptotic pathway in human colon cancer cells. Cancer Res 62: 6323-6328, 2002 .
18. Martinou JC, Desagher S and Antonsson B: Cytochrome c release from mitochondria: all or nothing. Nat Cell Biol 2: E41-E43, 2000

19. Livak KJ and Schmittgen TD: Analysis of relative gene expression data using real-time quantitative PCR and the 2(-Delta Delta C(T)) method. Methods 25: 402-408, 2001.

20. Fukuyama R, Ng KP, Cicek M, et al: Role of IKK and oscillatory NFkappaB kinetics in MMP-9 gene expression and chemoresistance to 5-fluorouracil in RKO colorectal cancer cells. Mol Carcinog 46: 402-413, 2007.

21. Tran NL, McDonough WS, Savitch BA, et al: Increased fibroblast growth factor-inducible 14 expression levels promote glioma cell invasion via Racl and nuclear factor-kappaB and correlate with poor patient outcome. Cancer Res 66: 9535-9542, 2006.

22. Konishi T, Sasaki S, Watanabe T, Kitayama J and Nagawa H: Overexpression of hRFI inhibits 5-fluorouracil-induced apoptosis in colorectal cancer cells via activation of NF-kappaB and upregulation of BCL-2 and BCL-XL. Oncogene 25: 3160-3169, 2006.

23. Tran NL, McDonough WS, Savitch BA, Sawyer TF, Winkles JA and Berens ME: The tumor necrosis factor-like weak inducer of apoptosis (TWEAK)-fibroblast growth factor-inducible 14 (Fn14) signaling system regulates glioma cell survival via NFkappaB pathway activation and BCL-XL/BCL-W expression. J Biol Chem 280: 3483-3492, 2005.

24. Polek TC, Talpaz M, Darnay BG and Spivak-Kroizman T: TWEAK mediates signal transduction and differentiation of RAW264.7 cells in the absence of Fn14/TweakR. Evidence for a second TWEAK receptor. J Biol Chem 278: 32317-32323, 2003.

25. Garg A and Aggarwal BB: Nuclear transcription factor-kappaB as a target for cancer drug development. Leukemia 16: 1053-1068, 2002.

26. Escarcega RO, Fuentes-Alexandro S, Garcia-Carrasco M, Gatica A and Zamora A: The transcription factor nuclear factorkappa B and cancer. Clin Oncol (R Coll Radiol) 19: 154-161, 2007.

27. Dolcet X, Llobet D, Pallares J and Matias-Guiu X: NF- $\kappa B$ in development and progression of human cancer. Virchows Arch 446: 475-482, 2005.

28. Reed JC: Bcl-2 family proteins: regulators of apoptosis and chemoresistance in hematologic malignancies. Semin Hematol 34: 9-19, 1997.

29. Zhu H, Guo W, Zhang L, et al: Bcl-XL small interfering RNA suppresses the proliferation of 5-fluorouracil-resistant human colon cancer cells. Mol Cancer Ther 4: 451-456, 2005.

30. Lei X, Huang Z, Zhong M, Zhu B, Tang S and Liao D: Bcl-XL small interfering RNA sensitizes cisplatin-resistant human lung adenocarcinoma cells. Acta Biochim Biophys Sin 39: 344-350, 2007.

31. Whitsett TG, Cheng E, Inge L, et al: Elevated expression of Fn14 in non-small cell lung cancer correlates with activated EGFR and promotes tumor cell migration and invasion. Am J Pathol 181: 111-120, 2012.

32. Willis AL, Tran NL, Chatigny JM, et al: The fibroblast growth factor-inducible 14 receptor is highly expressed in HER2-positive breast tumors and regulates breast cancer cell invasive capacity. Mol Cancer Res 6: 725-734, 2008.

33. Merkhofer EC, Cogswell P and Baldwin AS: Her2 activates NF-kappaB and induces invasion through the canonical pathway involving IKKalpha. Oncogene 29: 1238-1248, 2010.

34. Tsai CM, Yu D, Chang KT, et al: Enhanced chemoresistance by elevation of p185neu levels in HER-2/neu-transfected human lung cancer cells. J Natl Cancer Inst 87: 682-684, 1995.

35. Gusterson BA, Gelber RD, Goldhirsch A, et al: Prognostic importance of c-erbB-2 expression in breast cancer. International (Ludwig) Breast Cancer Study Group. J Clin Oncol 10: 1049-1056, 1992.

36. Munoz-Garcia B, Martin-Ventura JL, Martinez E, et al: Fn14 is upregulated in cytokine-stimulated vascular smooth muscle cells and is expressed in human carotid atherosclerotic plaques: modulation by atorvastatin. Stroke 37: 2044-2053, 2006.

37. Ortego M, Bustos C, Hernandez-Presa MA, et al: Atorvastatin reduces NF-kappaB activation and chemokine expression in vascular smooth muscle cells and mononuclear cells. Atherosclerosis 147: 253-261, 1999.

38. Muerkoster S, Wegehenkel K, Arlt A, et al: Tumor stroma interactions induce chemoresistance in pancreatic ductal carcinoma cells involving increased secretion and paracrine effects of nitric oxide and interleukin-1beta. Cancer Res 64: 1331-1337, 2004. 